\title{
E1 G1000 de Madrid: un ejemplo de sorteo y deliberación como complemento de la representación
}

\author{
Madrid G1000:An Example of Drawing and Deliberation \\ as a Complement to Representation
}

FERNANDO NAVARRO

Universttat Jaume I de CAStelló

Artículo recibido: 22 junio 2017

Solicitud de revisión: 27 junio 2017

Artículo aceptado: 12 julio 2017

La crisis de legitimidad del sistema de partidos tradicional que cristalizó en España el 15 de mayo de 2011 ${ }^{1}$ (Cano y Alemán, 2017; Tormey y Feenstra, 2015) ha provocado una enorme revisión teórica sobre si esa crisis tuvo más que ver con un cuestionamiento del modelo democrático de representación (Tormey, 2015a), o si se reducía a una deslegitimación de los dos partidos tradicionales en la historia reciente de España, el Partido Popular y el Partido Socialista. En otros números de Recerca ya se problematizó sobre el origen económico y social de esa crisis de legitimidad de las instituciones (Feenstra y Dekker, 2015) y se trazó la hipótesis sobre si las nuevas subjetivaciones individualizadas propias de la sociedad de la información, la globalización y la crisis de los grandes partidos de masas podía suponer una crisis del sistema de representación, o si solo exigía una nueva rotación de élites representantes (Tormey, 2015b). Si la explicación residiese en la segunda hipótesis, el surgimiento de nuevas opciones de representación como Ciudadanos o Podemos deberían haber cerrado esa crisis. En cambio, el último barómetro del cis del mes de abril señala que todavía un $68 \%$ de la población considera que la situación política es mala o muy mala; el $28 \%$ opina que la situación es peor que hace un año

1 El 15 de mayo de 2011 en España comenzaron las movilizaciones del 15M, protestas multitudinarias en las principales ciudades del país protestando contra la crisis económica y el sistema de representación. 
y el $55 \%$ considera que nada ha cambiado; solo un $12,2 \%$ cree que la situación ha mejorado con la entrada de nuevos representantes. ${ }^{2}$

Si la crisis del sistema de partidos no se ha resuelto con la incorporación de nuevos representantes, quizá la solución se deba encontrar mediante el diseño de nuevos mecanismos de participación ciudadana en la vida pública. Esa es la tesis defendida por David Van Reybrouck en Contra las elecciones (2017), una defensa del sistema de representación por sorteo en lugar de por elección ciudadana que ha servido de inspiración a una de las experiencias más novedosas en la democracia española: el G1000 de Madrid, organizado el pasado 4 de marzo desde la Concejalía de Participación Ciudadana y Gobierno Abierto.

El proceso de participación ciudadana G1000 fue una idea surgida en junio de 2011 en Bélgica con un primer manifiesto que lanzaron 17 personas ante la crisis de gobernabilidad que vivía el país en aquel momento. ${ }^{3}$ Con origen en la sociedad civil y apoyo de algunas personas de la vida pública belga, de académicos (incluido el propio Van Reybrouck) y estudiantes universitarios, la iniciativa proponía reunir a 1000 personas escogidas por sorteo para deliberar sobre temáticas fundamentales de la vida pública, con la convicción de que la democracia deliberativa podía ser más útil para el bien común que sistemas de representación clásicos (Fishkin, 2011). El 11 de noviembre de 2011 se organizaron 81 mesas de 10 personas cada una para deliberar sobre seguridad social, redistribución de la riqueza e inmigración. Las conclusiones de ese trabajo fueron trasladadas en una fase posterior al Parlamento de Flandes, al de Valonia y al Parlamento Federal Belga.

A partir de ese ejemplo, el Ayuntamiento de Madrid organizó el pasado 4 de marzo una experiencia similar, aunque esta vez la iniciativa surgía desde la esfera institucional -Ayuntamiento de Madrid- y se incluía dentro del proceso de participación ciudadana Decide Madrid: el G1000. Antes de esa fecha, personas de distintos distritos, sexos y edades fueron seleccionados de manera aleatoria para participar en el proceso de deliberación, centrado en discutir sobre el modelo de ciudad para Madrid y sobre el objetivo de los 100 millones de partida para presupuestos participativos.

La iniciativa del G1000 de Madrid fue una iniciativa coordinada por el laboratorio de experimentación democrática Medialab-Prado, que coordina

2 Cis (2017) Barómetro de opinión pública del Centro de Investigaciones Sociológicas de abril de 2017. [Disponibleen: http://datos.cis.es/pdf/Es3173mar_A.pdf, recuperado el 15 de junio de 2017]

3 Bélgica vivió entre 2010 y 2011 una crisis de gobernabilidad que tuvo al país sin gobierno durante 540 días debido a la incapacidad del Parlamento de nombrar a un nuevo Ejecutivo, incluso después de una repetición electoral. 
Yago Bermejo y que está acompañando todos los procesos del Ayuntamiento de Madrid. En una entrevista concedida para esta investigación el 17 de mayo de 2017 [parte de la cual reproducimos a continuación], Yago Bermejo define el G1000 como «una experiencia de democracia deliberativa a gran escala que tiene por objetivo desarrollar un debate sobre las propuestas para los Presupuestos Participativos del Ayuntamiento de Madrid y que tiene por objetivo sentar el comienzo de espacios de democracia deliberativa en la ciudad».

Recerca: -El paradigma de la democracia deliberativa ha ganado respaldos en las últimas décadas como mecanismo de legitimación y racionalización de la política, como un procedimiento de justificación ante la opinión pública del uso que se realiza del poder político (Habermas, 1998: 376). Para el pensamiento habermasiano, solo es justa o legítima aquella acción en la que todos los afectados por dicha política permite la participación en igualdad de condiciones de todos los afectados, una «situación ideal de habla» caracterizada por una distribución simétrica de los derechos y oportunidades, es decir, por la ausencia de barreras que obstruyan la comunicación (García Marzá, 1998). En el caso del G1000 de Madrid, ¿cuál fue la metodología seguida para alcanzar esa «situación ideal de habla»?

Yago Bermejo: -Durante la jornada del 4 de marzo, aproximadamente 300 personas se reunieron en el G1000 de Madrid y desarrollaron un proceso de deliberación en torno a 2 bloques: el primer bloque fue una toma de contacto abierto donde se discutía sobre posibles propuestas para los Presupuestos Participativos y se redactaban enunciados sobre cuestiones abiertas relacionadas con movilidad, con la limpieza... sin límite de propuestas y solo con una moderación que facilitaba la comunicación, pero que no influía en la deliberación. En la segunda fase se categorizaron las ideas de cada persona por colores que correspondían a áreas temáticas y se agrupaban las personas con enunciados parecidos; en esta segunda fase continuaba la deliberación y se trataban de consensuar propuestas en cada ámbito temático. Pero sin forzar ningún consenso, solo se facilitaba; si en una mesa temática había diferencias y salía más de una propuesta, se fragmentaba la mesa y continuaba la deliberación en cada idea. Finalmente se escogía una portavocía por mesa encargada de redactar la propuesta y subirla a la plataforma de Decide Madrid.

Recerca: -En el proceso de racionalización de la política del que habla $\mathrm{Ha}$ bermas, juega un papel fundamental la inclusión de todos los afectados 
en el proceso deliberativo que ha de desarrollarse para alcanzar consensos intersubjetivos (Habermas, 1998: 394). Es en este paso donde el G1000 constituye una novedad al apostar por la aleatoriedad como mecanismo de selección. ¿De qué forma el G1000 incorporó en su procedimiento a todos los afectados por la política de la ciudad de Madrid?

Yago Bermejo: -Se establecieron 30 mesas de 10 personas cada una que, en cómputo global, debían ser representativas de toda la ciudad. Se contrató a una empresa de demoscopia que realizó un estudio estadístico sobre la composición de Madrid por género, edades, condición socioeconómica, recuento de voto... A partir de ese estudio se desarrolló un desglose de los distintos perfiles que era necesario rellenar en cada franja social para que las 1000 personas fuesen la muestra lo más representativa posible de la ciudad de Madrid. A partir de esa tabla estadística, se desarrolló una estrategia de contacto en la calle (en centros comerciales, centros de salud, en distintos barrios, distritos...) y se fueron completando los distintos perfiles necesarios para completar la muestra.

Recerca: -Una de las críticas clásicas que desde las concepciones del elitismo democrático (Schumpeter, 1983) se ha dirigido a las propuestas de democracia participativa es la incapacidad de la ciudadanía para abstraerse de los intereses particulares o de la incapacidad de perseguir intereses comunes. Schumpeter llega a afirmar que el «ciudadano común desciende a un nivel inferior de prestación mental tan pronto como penetra en el campo de la política, se vuelve primitivo, se vuelve irracional». ¿Cuál es la respuesta del planteamiento del G1000 frente a esa defensa de la política como una tarea de élites?

Yago Bermejo: - Precisamente, una de las ideas que nos llevaron a plantear el G1000 fue el hecho de que los nuevos mecanismos de participación estaban generando una burbuja de mecanismos de participación, donde fundamentalmente participaba una minoría de la población, personas habituadas a la participación, sospechamos que simpatizantes de Ahora Madrid $^{4}$ o activistas con cierta experiencia en política municipal; pero había mucha gente que ni siquiera conocía los nuevos sistemas de participación que estábamos desarrollando. Por eso, uno de los objetivos del G1000 era lograr situar la participación en la agenda mediática, y creo que logramos cierto impacto en medios de comunicación. ${ }^{5}$ Además,

4 Ahora Madrid es la candidatura que gobierna el Ayuntamiento de Madrid desde las elecciones municipales del 24 de mayo de 2015.

5 Madrid celebra el G1000, una cumbre ciudadana (4 de marzo de 2017). ABC. (Recuperado en: http:// www.abc.es/espana/abci-madrid-celebra-g1000-cumbre-5347730965001-20170304010005_video. html, consultado el 19 de junio de 2017) 
teníamos la convicción de que cualquier persona podía discutir sobre la política de su ciudad, desarrollar ideas y presentar propuestas para los Presupuestos Participativos con la misma calidad que las propuestas de personas con más experiencia en política. Después del proceso de recogida de apoyos en el sistema de Decide Madrid $^{6}$ y de la evaluación por parte del funcionariado del Ayuntamiento de Madrid, hay dos propuestas surgidas del primer G1000 que han pasado a la fase de las votaciones finales para escoger el destino de los 100 millones de euros del Ayuntamiento de Madrid.

Recerca: -Además de como mecanismo de legitimación de los sistemas representativos, en los últimos años han proliferado las propuestas de superación de la diferenciación misma entre representantes y representados (Manin, 2006:247-303). Entre esas propuestas, destaca la obra Contra las elecciones (Van Reybrouck, 2017), quien llega a proponer una sustitución completa de la representación por el sorteo ${ }^{7}$ y la democracia deliberativa. ¿Iniciativas como el G1000 tienen vocación o intención de ir ocupando más instancias de toma de decisiones, más allá del método de participación en los presupuestos participativos?

Yago Bermejo: -El G1000 de Madrid es una iniciativa de Medialab-Prado, ${ }^{8}$ que es un laboratorio de experimentación democrática que probamos nuevos mecanismos de participación para ir innovando en la gestión política tradicional. Yo no tengo una certeza de cuál será el modelo que se abrirá paso en las próximas décadas, creo que eso exige que se vayan produciendo nuevas iniciativas, análisis y estudios que vayan comparando resultados y prescribiendo procedimientos y apuestas políticas valientes por la participación.

6 El sistema de Decide Madrid es el proyecto de Presupuestos Participativos del Ayuntamiento de Madrid. Se divide en 4 fases: una primera fase de presentación de proyectos (donde se introdujo como novedad en 2017 el G1000), una segunda fase de apoyos telemáticos, una tercera fase de evaluación de los proyectos más apoyados por parte del funcionariado del Ayuntamiento de Madrid para comprobar viabilidad económica y marco competencial para poder desarrollar la propuesta, $\mathrm{y}$ una tercera fase de votación. El presupuesto total de Decide Madrid es de 100 millones de euros, 30 millones para iniciativas que afecten a toda la ciudad y 70 millones repartidos por distritos (las propuestas del G1000 debían ser para toda la ciudad). Más información en: https://decide.madrid.es/

7 El sorteo como mecanismo democrático tiene su origen en la Antigua Grecia, pero se perdió su uso con la recuperación de la democracia moderna a favor de la elección representativa. Sin embargo la crisis de los sistemas representativos han devuelto cierto interés en el sorteo (Manin, 2006; Van Reybrouck, 2017; Avaro, 2014)

8 Medialab-Prado se definen a sí mismos en su página web como «un laboratorio ciudadano de producción, investigación y difusión de proyectos culturales que explora las formas de experimentación y aprendizaje colaborativo que han surgido de las redes digitales». Más información en: http://medialabprado.es/ 


\section{CONCLUSIONES}

La experiencia del G1000 se encuadra dentro de otras apuestas democráticas por la deliberación y el sorteo: en Islandia se constituyó en 2011 una Asamblea Constituyente Nacional compuesta por ciudadanía escogida al azar para preparar una nueva Constitución con un proceso deliberativo durante 4 meses $^{9}$ y, más recientemente, en Irlanda se creó un convención mixta compuesta por representantes públicos y ciudadanos escogidos al azar para discutir sobre la reforma de la Constitución. ${ }^{10}$ Por eso, la reflexión sobre los retos de la democracia en el siglo xxi puede encontrar en el caso del G1000 una experiencia para pensar cómo avanzar.

Además, el G1000 innova dentro de las propuestas de democracia participativa tratando de corregir el sesgo que provocan otros mecanismos de participación que sólo logran movilizar a un estrato muy reducido de la población, apostando por el sorteo para garantizar la representatividad de los participantes del proceso, mediante correcciones para cubrir los distintos perfiles que componen una sociedad dada, en este caso la ciudad de Madrid.

Después de prácticamente tres siglos de paradigma ininterrumpido del clásico sistema de representación, el nuevo contexto de la globalización y la sociedad-red, la desafección con las viejas élites políticas y la aparición de nuevos mecanismos de participación obligan a repensar, evaluar y mejorar los convencionales modelos de democracia. La propuesta del G1000 de Madrid como mecanismo de participación ciudadana, de deliberación y de uso del sorteo como mecanismo democrático supone un intento por tratar de buscar una salida a la crisis de legitimidad del sistema de participación español, común a otros países de Europa y América.

9 Islandia y la nueva Constitución: la participación ciudadana está siendo parada por los políticos. Periódico del Bien Común. (Recuperado en: http://www.periodicodelbiencomun.com/propuestasproyectos/islandia-y-una-nueva-constitucion-la-participacion-ciudadana-esta-siendo-frenada-por-lospoliticos/, consultado el 19 de junio de 2017)

10 Ortega,Andrés (3 de abril de2014). Democracia deliberativa: un buen ejemplo irlandés. (Recuperado de: http://www.eldiario.es/zonacritica/Democracia-deliberativa-buen-ejemplo-irlandes_6_245735436.html, consultado el 19 de junio de 2018) 


\section{REFERENCIAS BIBLIOGRÁFICAS}

Avaro, D. (2014): «El sorteo y la democracia: ¿son posibles nuevos horizontes de complementariedad con la representación", Revista Encrucijada Americana, 2, pp. 115-130.

Cano, G. y Alemán, J. (2017): Del desencanto al populismo: encrucijada de una época, Madrid, Nuevos emprendimientos editoriales.

DekKer, P. y Feenstra, R. (2015): «Activism and civil society: broadening participation and deepening democracy», Recerca. Revista de pensament crític $i$ anàlisi, 17: pp. 7-15

Fishкin, J. (2011): When the people speak: Deliberative democracy and public consultation, Oxford, Oxford University Press.

GarCíA-MARZÁ, D. (1990): Democracia participativa: entre utopia i realitat, Recerca, 5, pp. 70-78

Habermas, J. (1998): Facticidad y validez, Madrid, Trotta.

MANIN, B. (2006): Los principios del gobierno representativo, Madrid,Alianza Editorial

SCHUMPETER, M. (1983): Capitalismo, socialismo y democracia, Barcelona, Orbis.

Tormey, S. (2015a): The End of Representative Politics, Cambridge, Polity Press.

- (2015b): «Democracy will never be the same again: $21^{\text {st }}$ Century Protest and the Transformation of Politics», Recerca. Revista de pensament crític $i$ anàlisi, 17, pp.107-128.

Tormey, S. y Feenstra, R. (2015): «Reinventing the polical party in Spain: the case of 15M and the Spanish mobilisations», Policy Studies, 36.

Van Reybrouck, D. (2017): Contra las elecciones. Madrid,Taurus. 